\title{
Self-interacting dark matter and monochromatic lines
}

\section{Takashi Toma*}

Laboratoire de Physique Théorique, CNRS,

Univ. Paris-Sud, Université Paris-Saclay, 91405 Orsay, France

E-mail: takashi.toma@th.u-psud.fr

\begin{abstract}
The recent observation of the cluster Abell3827 implies the offset between the center of the dark matter sub-halo and visible stars. This offset can be interpreted by large self-interaction of dark matter. We consider a simple pseudo-scalar dark matter model in which allowed parameter space is investigated by taking into account some relevant constraints. We see that dark matter with $\mathrm{keV}$ to $\mathrm{MeV}$ scale mass is favored in order to obtain large self-interacting cross section and monochromatic line signals simultaneously. Moreover, detectability of such a light dark matter particle by future direct detection experiments with superconductors is discussed.
\end{abstract}

The 11th International Workshop Dark Side of the Universe 2015

14-18 December 2015

Kyoto, Japan

${ }^{*}$ Speaker. 


\section{Introduction}

There are many evidences for the existence of dark matter such as rotation curves of spiral galaxies, CMB observation, gravitational lensing effects, large scale structure of the universe and collision of the bullet cluster. Although the existence of dark matter is crucial from these evidences, the mass and interactions of dark matter are not known yet and we know only the current dark matter energy density is $\Omega h^{2}=0.1199 \pm 0.0081$ at $1 \sigma$ confidence level [1].

The cluster Abell3827 is locally made of four galaxies and the recent observation of this cluster has claimed that the offset $\Delta=1.62 \mathrm{kpc}$ between the center of the dark matter sub-halo and stars with $3.3 \sigma$ significance [2]. This observation may be interpreted by large self-interaction of dark matter. According to two different analyses, the required self-interaction of dark matter to explain the offset is estimated as $\sigma / m \sim 1.5 \times 10^{-4} \mathrm{~cm}^{2} / \mathrm{g}$ [2] and $\sigma / m \sim 1.5 \mathrm{~cm}^{2} / \mathrm{g}$ [3] respectively where $m$ is the dark matter mass. One can see that these values are very large if the typical Weakly Interacting Massive Particles (WIMPs) with electroweak scale mass are assumed as dark matter since the typical value for WIMPs is given by $\sigma / m \lesssim 10^{-11} \mathrm{~cm}^{2} / \mathrm{g}$. Thus one needs a mechanism to achieve such a large self-interacting cross section like strongly interacting massive particles, Sommerfeld enhancement with a light mediator, hidden sector dark matter.

On the other hand, monochromatic X-ray or gamma-ray lines generated by dark matter annihilations or decays can be a smoking-gun signature of dark matter. For instance, the $3.5 \mathrm{keV} \mathrm{X-ray}$ line has been observed in Perceus and Andromeda galaxies with $\sim 4 \sigma$ significance $[4,5]$, and this line may be interpreted by dark matter annihilations or decays. ${ }^{1}$

Here we consider a simple dark matter model in which we introduce a complex scalar field written by scalar and pseudo-scalar components, and dark matter is identified as the pseudo-scalar particle. The self-interacting cross section and annihilation cross section into two photons are computed, and correlation between them is discussed together with some constraints from astrophysical observations, collider experiments, X-ray and gamma-ray observations and coupling perturbativity.

\section{The Model}

We consider a simple model extended by a complex scalar $\Phi$ with a global $U(1)$ symmetry [8]. The general renormalizable potential for the complex scalar field $\Phi$ respecting the global $U(1)$ symmetry is given by

$$
\mathscr{V}_{\Phi}=-\mu^{2}|\Phi|^{2}+\frac{\lambda}{4}|\Phi|^{4}
$$

where $\mu^{2}$ is the bare mass of $\Phi$ and $\lambda$ is the quartic coupling. The quartic coupling with the SM Higgs doublet which induces mixing between the SM Higgs boson and the scalar component of $\Phi$ is neglected here. Note that the self-interacting coupling $\lambda$ is necessary allowed for scalar fields, while a mediator is needed for fermions to induce four fermi interaction effectively as a self-coupling.

\footnotetext{
${ }^{1}$ Note that such X-ray line excess is not observed from the other galaxies such as dwarf stacked galaxies [6] and Milky Way [7].
} 
After the spontaneous symmetry breaking of the global $U(1)$ symmetry, the scalar potential can be rewritten with $\Phi=v+\frac{s+i a}{\sqrt{2}}\left(v=\langle\Phi\rangle=\sqrt{\frac{2}{\lambda}} \mu\right)$ as

$$
\mathscr{V}_{\Phi}=\frac{m_{s}^{2}}{2} s^{2}+\frac{m_{s}}{2} \sqrt{\frac{\lambda}{2}} s\left(s^{2}+a^{2}\right)+\frac{\lambda}{16}\left(s^{2}+a^{2}\right)^{2}
$$

where the scalar mass is given by $m_{s}=\sqrt{2} \mu=\sqrt{\lambda}$. Although the Goldstone boson $a$ is massless, a small mass is expected to be generated by anomaly of the global $U(1)$ symmetry breaking at a high energy scale [9]. In the following, we assume that the Goldstone boson $a$ has a small mass $\left(m_{a} \ll m_{s}\right)$ and identify as dark matter since there is no any operator inducing decay of the pseudoscalar particle.
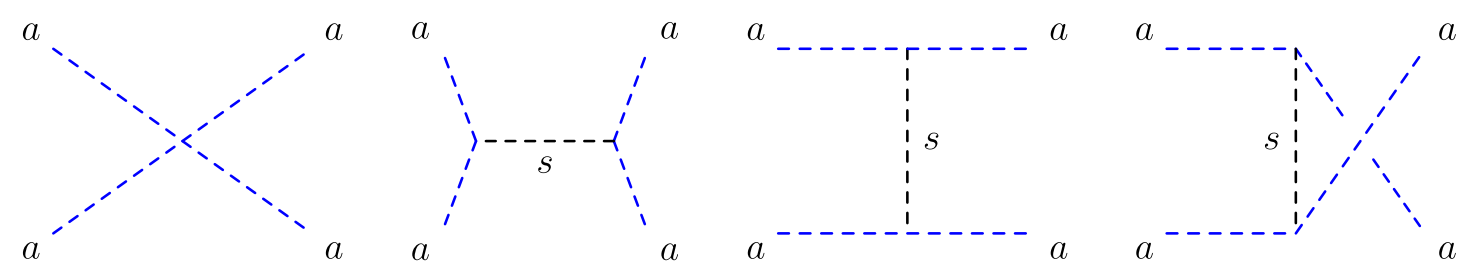

Figure 1: Relevant diagrams for dark matter self-interacting cross section.

The dark matter self-interacting process $a a \rightarrow a a$ is induced by the interactions in the scalar potential Eq. (2.2). The relevant diagrams for dark matter self-interaction is shown in FIg. 1, and the self-interacting cross section can easily be computed by

$$
\frac{\sigma_{a a}}{m_{a}}=\frac{\lambda^{2} m_{a}}{32 \pi\left(m_{s}^{2}-4 m_{a}^{2}\right)^{2}} \simeq \frac{\lambda^{2} m_{a}}{32 \pi m_{s}^{4}},
$$

where $m_{a} \ll m_{s}$ is assumed. As one can see from Eq. (2.3), the self-interacting cross section is proportional to the dark matter mass squared $\left(\sigma_{a a} \propto m_{a}^{2}\right)$. This behaviour is different from typical cold dark matter such that cross sections of a non-relativistic particle is usually proportional to inverse of the squared mass. This different property is due to the pseudo-Goldstone boson dark matter in our model. This behaviour is similar with the $W W$ scattering in the SM.

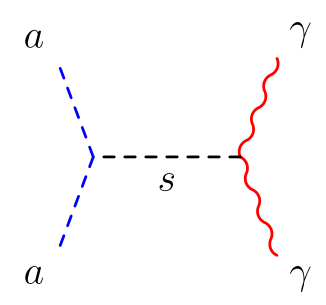

Figure 2: Relevant diagram for dark matter annihilation into two photons.

In addition to the renormalizable interactions discussed above, we consider the dimension 5 effective operator

$$
\mathscr{L}_{s \gamma \gamma}=\frac{s}{\Lambda} F_{\mu v} F^{\mu v}
$$


where $F_{\mu v}=\partial_{\mu} A_{v}-\partial_{v} A_{\mu}$ is the electromagnetic field strength. Monochromatic photons are generated by dark matter annihilations through the effective operator as shown in Fig. 2. This operator is expected to be induced at loop level by running of charged particles. We can compute the annihilation cross section of dark matter into two photons $(a a \rightarrow \gamma \gamma)$, which is give by

$$
\sigma v_{\gamma \gamma}=\frac{\lambda m_{a}^{2} m_{s}^{2}}{\pi \Lambda^{2}\left(m_{s}^{2}-4 m_{a}^{2}\right)^{2}} .
$$

Combining Eq. (2.3) and (2.5), we can eliminate the self-coupling $\lambda$, and express the annihilation cross section as a function of $\sigma_{a a} / m_{a}$,

$$
\sigma v_{\gamma \gamma}=\frac{4 \sqrt{2} m_{a}^{3 / 2}}{\Lambda^{2} \sqrt{\pi}} \sqrt{\frac{\sigma_{a a}}{m_{a}}} \simeq 1.3 \times 10^{-33}\left(\frac{100 \mathrm{TeV}}{\Lambda}\right)^{2}\left(\frac{m_{a}}{3 \mathrm{keV}}\right)^{3 / 2} \sqrt{\frac{\sigma_{a a} / m_{a}}{1 \mathrm{~cm}^{2} / \mathrm{g}}} \mathrm{cm}^{3} / \mathrm{s}
$$

This equation shows one-to-one correspondence between the self-interacting cross section and the annihilation for the process $a a \rightarrow \gamma \gamma$.

In this model, the dark matter relic abundance cannot be accommodated by the standard freezeout mechanism since the possible annihilation channels are quite restricted $\left(\gamma \gamma, v \bar{v}, e^{+} e^{-}\right)$and the cross section is suppressed by the mass of the mediator (the SM Higgs boson) or the small Yukawa coupling. However, we may be able to satisfy the dark matter relic abundance by freeze-in mechanism via the small mixing between the SM Higgs boson and the scalar $s$ we neglected, or by extending the model to annihilate hidden sector particles for example.

\section{Numerical Analyses}

\subsection{The constraints}

Here we consider some constraints relevant to the model. First, the dimension 5 operator introduced in Eq. (2.4) may cause the production of the scalar mediator particle $s$ inside stars through photons in thermal equilibrium. The helium burning lifetime of the stars is shortened because of this non-standard interaction of photon. This gives a constraint on the cut-off scale $\Lambda$ (HB bound). Second, the process of mono-photon plus missing energy $e^{+} e^{-} \rightarrow \gamma_{S}$ can be induced at collider experiments, which is strongly constrained by Anomalous Single Photon (ASP) [10] and Large Electron-Positron (LEP) experiments [11]. ${ }^{2}$ Third, the annihilation cross section for $a a \rightarrow \gamma \gamma$ is constrained by $\mathrm{X}$-ray and gamma-ray experiments. The wide energy range from $\mathrm{keV}$ to $\mathrm{GeV}$ is covered by HEAO-1, INTEGRAL, COMPTEL, EGRET, FERMI [12]. Finally, the self-coupling of dark matter $\lambda$ should be perturbative. We take the benchmark of the perturbativity as $\lambda<4 \pi$.

\subsection{Combining the self-interaction and the monochromatic lines}

We combine the self-interacting cross section and the annihilation cross section for monochromatic lines taking into account all the relevant constraints. The numerical results are shown in Fig. 3 in the plane of $\left(m_{a}, \Lambda\right)$ where the mass ratio between $m_{a}$ and $m_{s}$ is fixed to be $m_{s} / m_{a}=10$ and the self-interacting cross section is taken as $\sigma_{a a} / m_{a}=1.7 \times 10^{-4} \mathrm{~cm}^{2} / \mathrm{g}$ (left panel) and

\footnotetext{
${ }^{2}$ If the decay length of the scalar for $s \rightarrow \gamma \gamma$ is shorter than the size of the detector, the scalar $s$ can decay inside the detector and three photons are emitted. In this case, the constraint is not applied.
} 
$\sigma_{a a} / m_{a}=1.5 \mathrm{~cm}^{2} / \mathrm{g}$ (right panel). The dark matter mass is bounded from above by the perturbativity of the self-coupling $\lambda<4 \pi$, and bounded from below by the HB bound. The lower $\Lambda$ is excluded by the X-ray and gamma-ray experiments. Although the collider constraint is not shown here, some parameter space of $\Lambda$ lower than $10^{4} \mathrm{GeV}$ is excluded. The gamma-ray constraint is much stronger than the collider constraint. As one can see from the results, the allowed parameter range is given by

$$
\begin{aligned}
10 \mathrm{keV} \lesssim m_{a} \lesssim 10 \mathrm{MeV}, \quad \Lambda \gtrsim 10^{5} \mathrm{GeV}, & \text { for } \quad \sigma_{a a} / m_{a}=1.7 \times 10^{-4} \mathrm{~cm}^{2} / \mathrm{g}, \\
10 \mathrm{keV} \lesssim m_{a} \lesssim 1 \mathrm{MeV}, \quad \Lambda \gtrsim 10^{6} \mathrm{GeV}, & \text { for } \quad \sigma_{a a} / m_{a}=1.5 \mathrm{~cm}^{2} / \mathrm{g} .
\end{aligned}
$$
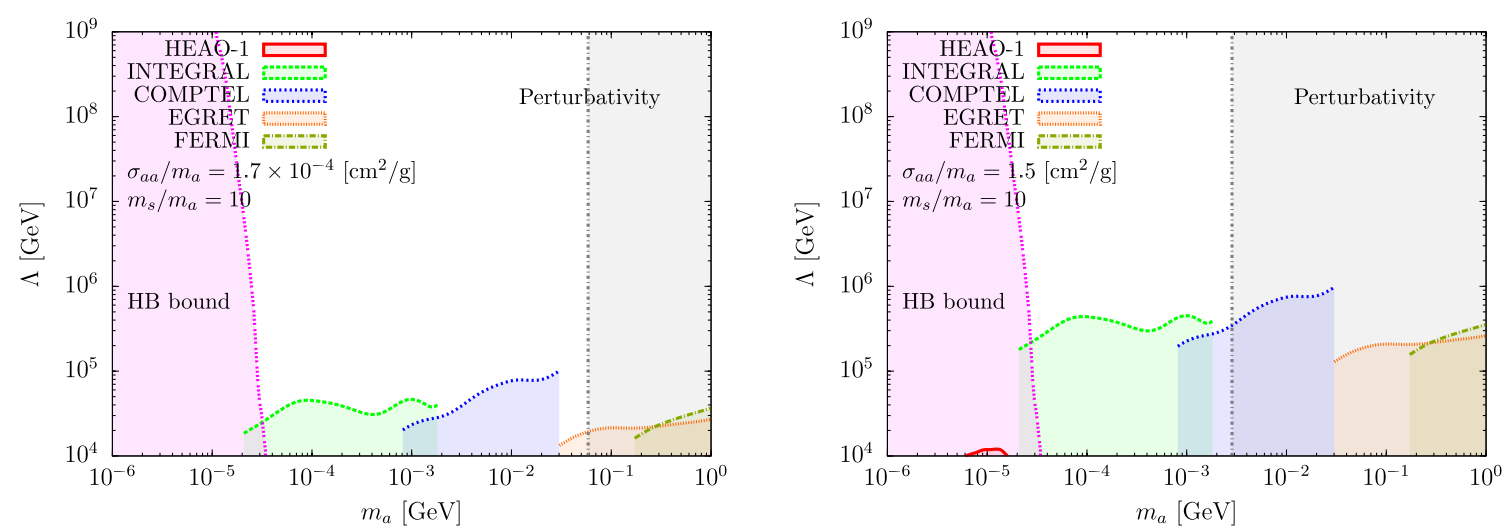

Figure 3: Numerical result obtained by combining the self-interacting cross section and the annihilation cross section for monochromatic photons. The colored region is excluded by the constraints.

In the case of fixing the dark matter mass at $m_{a}=3.5 \mathrm{keV}$ for the $\mathrm{X}$-ray excess, the numerical result is shown in Fig. 4 where the self-interacting cross section is fixed as same as Fig. 3. The parameter $J_{\text {astro }}$ represents the astrophysical uncertainty which comes from the dark matter profile and integration along the line of sight. The allowed region of the mediator mass $m_{s}$ is sandwiched by the constraints of the coupling perturbativity and the HB bound as same as the above case with the non-fixed dark matter mass. One can see that the range of the cut-off scale $10 \mathrm{TeV} \lesssim \Lambda \lesssim$ $1000 \mathrm{TeV}$ is favored to fit to the $3.5 \mathrm{keV} \mathrm{X}$-ray excess depending on the value of the self-interacting cross section.

Non-detection of the $3.5 \mathrm{keV}$ X-ray excess from the other galaxies has been reported by some groups $[6,7]$. If this is true, the non-detection of the $\mathrm{X}$-ray excess gives a very strong constraint on the model. The upper bound for the annihilation cross section for the process $a a \rightarrow \gamma \gamma$ is set to be $\sigma v_{\gamma \gamma} \lesssim 2.5 \times 10^{-36} \mathrm{~cm}^{3} / \mathrm{s}$ from the stacked dwarf galaxies [6] and $\sigma v_{\gamma \gamma} \lesssim 7.9 \times 10^{-38} \mathrm{~cm}^{3} / \mathrm{s}$ from the Milky Way [7]. These limits completely exclude the interpretation of the X-ray excess by dark matter annihilation as shown in Fig. 5 where the NFW profile is assumed. However one should note that the limits strongly depend on the assumption of the dark matter profile. If a cored dark matter profile is assumed such as Isothermal profile, the limits of the non-detection of the X-ray may be evaded. Moreover, other scenarios for example, interpretation of the X-ray excess due to an excited dark matter can evade this strong limit. 

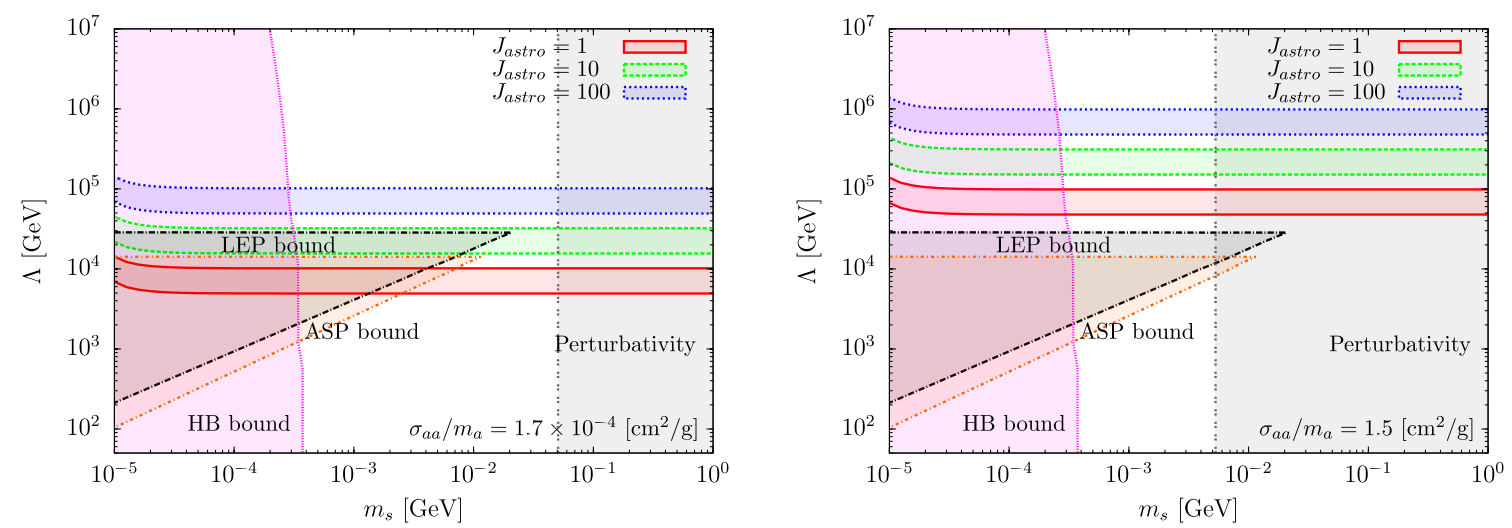

Figure 4: Numerical result obtained by combining the self-interacting cross section and the $3.5 \mathrm{keV} \mathrm{X}$-ray excess $\left(m_{a}=3.5 \mathrm{keV}\right)$ where $J_{\text {astro }}$ stands for the astrophysical uncertainty.
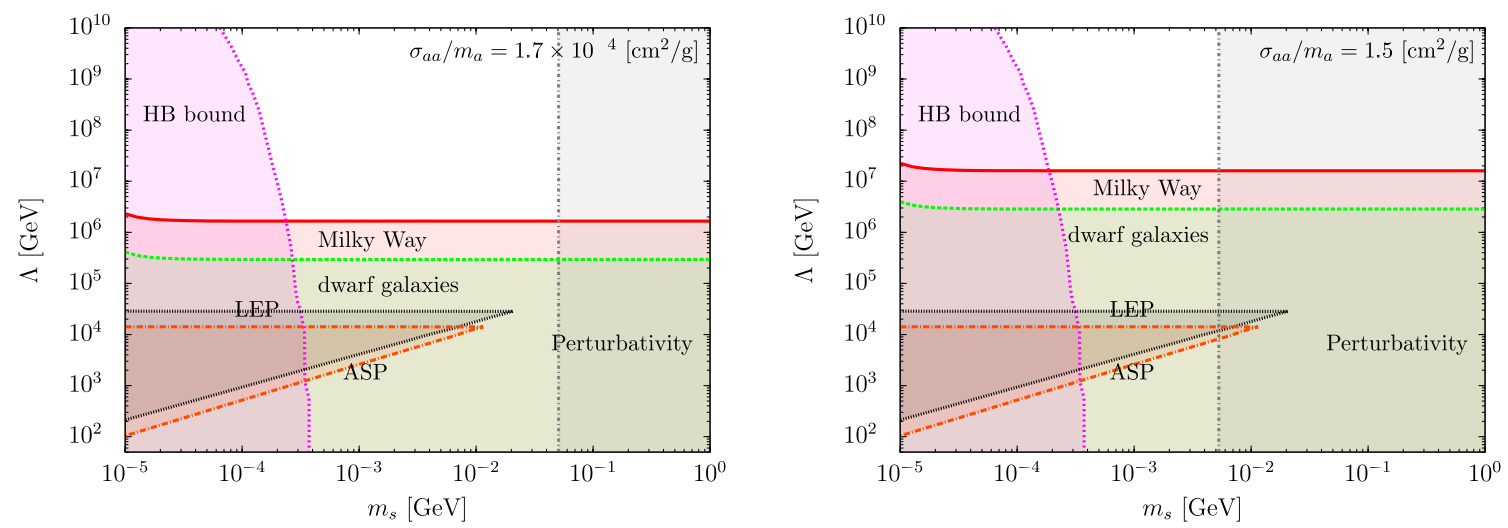

Figure 5: Limits of non-detection of the X-ray excess from the stacked dwarf galaxies and Milky Way where the NFW profile is assumed.

\subsection{Direct detection prospects}

We discussed $\mathrm{keV}$ to $\mathrm{MeV}$ scale dark matter to achieve the large self-interacting cross section. Such a light dark matter particle would be difficult to detect with direct detection experiments with nuclei. However we have chance to detect such a light dark matter by looking for elastic scattering event with electron. In particular, according to the recent paper [13], the target range of dark matter mass is from $10 \mathrm{keV}$ to $1 \mathrm{GeV}$, and the sensitivity of recoil energy for the elastic scattering can reach up to $\mathscr{O}(1) \mathrm{meV}$ by using superconductors. In our model, the mediator $s$ can basically couple with electron like $\mathscr{L}=g_{e} s \bar{e} e$ through the mixing with the SM Higgs boson while we neglected so far. Thus the elastic cross section with electron is computed by

$$
\sigma_{\mathrm{DD}}^{e}=\frac{\lambda^{2} g_{e}^{2}}{2 \pi m_{s}^{4}} \mu_{e a}^{2}\left(\frac{m_{s}^{2}}{4 m_{a}^{2}}\right)
$$

where $\mu_{e a} \equiv m_{a} m_{e} /\left(m_{a}+m_{e}\right)$ is the reduced mass. With this formula, the sensitivity for the coupling $g_{e}$ is roughly obtained as $g_{e} \gtrsim 10^{-7}$ by comparing the future sensitivity for the cross section 
in ref. [13] where the self-interacting cross section is fixed as $\sigma_{a a} / m_{a}=1.7 \times 10^{-4} \mathrm{~cm}^{2} / \mathrm{g}$ and $1.5 \mathrm{~cm}^{2} / \mathrm{g}$.

\section{Summary}

The recent observation of the cluster Abell3827 has shown the off-set between the center of the dark matter distribution and stars. This offset may be caused by large self-interaction of dark matter. We considered a simple pseudo-scalar dark matter model, and explored favored parameter space for the self-interaction of dark matter taking into account the relevant constraints including the HB bound, collider experiments, the coupling perturbativity and monochromatic lines coming from the dark matter annihilation. We found that the dark matter mass scale should be $\mathrm{keV}$ to $\mathrm{MeV}$ scale and such range of dark matter can be searched by the future direct detection experiments with superconductors.

\section{References}

[1] P. A. R. Ade et al. [Planck Collaboration], arXiv:1502.01589 [astro-ph.CO].

[2] R. Massey et al., Mon. Not. Roy. Astron. Soc. 449, no. 4, 3393 (2015) [arXiv:1504.03388 [astro-ph.CO]].

[3] F. Kahlhoefer, K. Schmidt-Hoberg, J. Kummer and S. Sarkar, Mon. Not. Roy. Astron. Soc. 452, no. 1, L54 (2015) [arXiv:1504.06576 [astro-ph.CO]].

[4] E. Bulbul, M. Markevitch, A. Foster, R. K. Smith, M. Loewenstein and S. W. Randall, Astrophys. J. 789, 13 (2014) [arXiv:1402.2301 [astro-ph.CO]].

[5] A. Boyarsky, O. Ruchayskiy, D. Iakubovskyi and J. Franse, Phys. Rev. Lett. 113, 251301 (2014) [arXiv:1402.4119 [astro-ph.CO]].

[6] D. Malyshev, A. Neronov and D. Eckert, Phys. Rev. D 90, 103506 (2014) [arXiv:1408.3531 [astro-ph.HE]].

[7] S. Riemer-Sorensen, arXiv:1405.7943 [astro-ph.CO].

[8] Y. Mambrini and T. Toma, Eur. Phys. J. C 75, no. 12, 570 (2015) [arXiv:1506.02032 [hep-ph]].

[9] E. Dudas, Y. Mambrini and K. A. Olive, Phys. Rev. D 91, 075001 (2015) [arXiv:1412.3459 [hep-ph]].

[10] M. Kleban and R. Rabadan, hep-ph/0510183.

[11] C. Hearty et al., Phys. Rev. D 39, 3207 (1989).

[12] R. Essig, E. Kuflik, S. D. McDermott, T. Volansky and K. M. Zurek, JHEP 1311, 193 (2013) [arXiv:1309.4091 [hep-ph]].

[13] Y. Hochberg, Y. Zhao and K. M. Zurek, Phys. Rev. Lett. 116, no. 1, 011301 (2016) [arXiv:1504.07237 [hep-ph]]. 